\title{
A Double Integral Containing the Modified Bessel Function: Asymptotics and Computation
}

\author{
By N. M. Temme
}

\begin{abstract}
A two-dimensional integral containing $\exp (-u-t) I_{0}(2 \sqrt{u t})$ is considered. $I_{0}(z)$ is the modified Bessel function and the integral is taken over the rectangle $0 \leqslant u \leqslant x, 0 \leqslant t \leqslant y$. The integral is difficult to compute when $x$ and $y$ are large, especially when $x$ and $y$ are almost equal. Computer programs based on existing series expansions are inefficient in this case. A representation in terms of the error function (normal distribution function) is discussed, from which more efficient algorithms can be constructed.
\end{abstract}

1. Introduction. We consider the Bessel function integral

$$
I(x, y)=\int_{0}^{x} \int_{0}^{y} e^{-u-t} I_{0}(2 \sqrt{u t}) d u d t
$$

where $x, y \geqslant 0$ and $I_{0}(z)$ is the modified Bessel function. Integrals of this type are encountered in many physical contexts; Goldstein [4] is a good example. A more recent paper of Lassey [6] gives various references on applications. In that paper, the computational problem is extensively discussed, not for (1.1), but for related integrals. The algorithms are based on series expansions in terms of modified Bessel functions or exponential polynomials of the type $\sum_{m=0}^{n} x^{m} / m$ !. These expansions are very convenient to implement, and they give efficient algorithms in the $(x, y)$-plane except in the neighborhood of the diagonal. So we do not consider the computational problem completely solved.

When $x$ and $y$ are large, and $|x-y|$ is small compared to $x$ and $y$, the integral (1.1) has a peculiar behavior. To see this, consider the well-known estimate

$$
I_{0}(z) \sim \frac{e^{z}}{\sqrt{2 \pi z}} \text { as } z \rightarrow \infty \text {. }
$$

It follows that in the outer parts of the quarter plane the integrand is exponentially small, except near the diagonal, where we can see a ridge with height $O(1 / \sqrt{x})$. This change in behavior causes the main problem for the computations based on the expansions mentioned earlier. We discuss an asymptotic expansion in terms of the error function (or normal distribution function) and related functions. This expansion is valid for $x, y$ large and it is uniformly valid with respect to $|x-y|$, which may vary in the interval $[0, \infty)$. The asymptotic expansion can be found in Goldstein

Received June 27, 1985; revised November 14, 1985.

1980 Mathematics Subject Classification. Primary 33A40, 41A60, 65D20.

Key words and phrases. Bessel function integral, uniform asymptotic expansion, incomplete gamma functions. 
[4]; see also Luke [7, p. 279]. We rederive the expansion by using a new integral representation, and we give error bounds for the remainders of the expansion. The asymptotic problem resembles that for cumulative distribution functions, for which the central limit theorem describes the role of the normal distribution function also. From the cited literature it follows that (1.1) in fact can be viewed as a distribution function.

In Lassey [6], a more general function is considered, in which the Bessel function of (1.1) exhibits an argument of the form $2 \sqrt{p u t}, p \geqslant 0$. It will be shown in Subsection 2.2 that our results for (1.1) can easily be generalized for this case, although the limiting form $p \rightarrow 1$ may cause some computational problems.

In the next section we give a new integral representation for $I(x, y)$, from which in subsequent sections an asymptotic expansion and numerical methods are derived. The estimates and error bounds for the remainders in the expansion show the asymptotic nature of the expansion and they give a sound basis for a numerical algorithm.

2. A New Integral Representation for (1.1). The result of this section is the representation

$$
\begin{gathered}
I(x, y)=x-\frac{1}{2} e^{-x-y}\left[(x+y) I_{0}(\xi)+\xi I_{1}(\xi)\right]+(y-x) F(x, y), \\
F(x, y)=\frac{1}{2} \tau \int_{\xi}^{\infty} e^{-\sigma t} f(t) d t \\
f(t)=e^{-t} I_{0}(t), \quad \xi=2 \sqrt{x y}, \quad \sigma=\frac{(\sqrt{y}-\sqrt{x})^{2}}{\xi}, \quad \tau=\frac{y-x}{\xi} .
\end{gathered}
$$

Before proving this, we make some remarks. $I(x, y)$ is symmetric in $x$ and $y$, so we can (and will) assume that $0 \leqslant x \leqslant y$. In (2.2), $\xi$ is the large asymptotic parameter and $\sigma$ is the uniformity parameter, $\sigma \geqslant 0$. The integral in (2.2) diverges on the diagonal. The factor $\tau$ in front of the integral makes $F(x, y)$ well defined at $x=y$. By partial integration a convergent integral (at $\sigma=0$ ) can be obtained, but our asymptotics does not need this. The term with the Bessel functions in (2.1) is exponentially small for large $\xi$, except when $x=y$ where it is $O(\sqrt{x})$. Also, since $F(x, y)$ is exponentially small off the diagonal, and since $I(x, \infty)=x$ (see Remark 2.2 below), it follows that (2.1) is a stable representation for computing $I(x, y)$ when we have a stable algorithm for $F(x, y)$.

2.1. Proof of (2.1), (2.2). To prove representation (2.1), (2.2), we first write

$$
\begin{gathered}
I(x, y)=x+(y-x) K(x, y)-e^{-x-y}\left[\frac{1}{2} \xi I_{1}(\xi)+x I_{0}(\xi)\right], \\
K(x, y)=\int_{0}^{x} e^{-(t+y)} I_{0}(2 \sqrt{t y}) d t .
\end{gathered}
$$

This can be verified by applying the operator $\partial^{2} / \partial x \partial y$ to both sides of (2.4) and using

$$
\frac{\partial K(x, y)}{\partial y}=-e^{-x-y} \sqrt{x / y} I_{1}(\xi), \quad \frac{\partial}{\partial x}\left[\sqrt{x / y} I_{1}(\xi)\right]=I_{0}(\xi) .
$$

Representation (2.4) also follows from more general results in Lassey [6], and it reduces the double integral (1.1) to the single integral plus a few modified Bessel 
functions. The function

$$
J(x, y)=1-K(x, y)=\int_{x}^{\infty} e^{-(t+y)} I_{0}(2 \sqrt{t y}) d t
$$

is considered in Goldstein [4] and summarized in Luke [7].

We proceed with (2.5) and replace the Bessel function by the contour integral

$$
I_{0}(2 \sqrt{t y})=\frac{1}{2 \pi i} \int_{L} e^{t / s+y s} \frac{d s}{s},
$$

where $L$ is a circle around $s=0$. This integral follows, for instance, from the generating function (Olver $[9$, p. 61])

$$
e^{z(t+1 / t) / 2}=\sum_{n=-\infty}^{\infty} I_{n}(z) t^{n},
$$

by using Cauchy's formula. By integrating (2.7) along the circle $|s|=\sqrt{t / y}$, the standard integral

$$
I_{0}(\xi)=\frac{1}{\pi} \int_{0}^{\pi} e^{\xi \cos \theta} d \theta
$$

follows. Substituting (2.7) into (2.5), we obtain

$$
K(x, y)=\frac{e^{-x-y}}{2 \pi i} \int_{L} \frac{e^{y s}\left(e^{x / s}-e^{x}\right)}{1-s} d s .
$$

When $L$ is a circle with radius less than unity, the term $-e^{x}$ in the numerator gives no contribution. Integrating (2.9) along the circle $|s|=\rho=\sqrt{x / y}$ (we assume temporarily $\rho<1$ ), we obtain

$$
K(x, y)=-\frac{e^{-x-y}}{2 \pi} \int_{0}^{\pi} e^{\xi \cos \theta} \frac{2 \rho(\rho-\cos \theta)}{\rho^{2}-2 \rho \cos \theta+1} d \theta .
$$

Write $2 \rho(\rho-\cos \theta)=\rho^{2}-2 \rho \cos \theta+1+\rho^{2}-1$. Then

$$
K(x, y)=F(x, y)-\frac{1}{2} e^{-x-y} I_{0}(\xi),
$$

where (2.8) is used, and

$$
F(x, y)=\frac{\left(1-\rho^{2}\right) e^{-x-y}}{2 \pi} \int_{0}^{\pi} \frac{e^{\xi \cos \theta}}{\rho^{2}-2 \rho \cos \theta+1} d \theta
$$

Combining (2.10) and (2.4) we obtain (2.1). Hence, we are finished when we have shown that (2.2) and (2.11) are the same. To prove this, we write

$$
F(x, y) / \tau=\frac{1}{2 \pi} \int_{0}^{\pi} \frac{e^{\xi(\cos \theta-\mu)}}{\mu-\cos \theta} d \theta,
$$

where $\tau$ is given in (2.3) and $\mu=\left(\rho^{2}+1\right) / 2 \rho=\sigma+1$. By assuming temporarily that $\mu$ is independent of $\xi$, we obtain with (2.8)

$$
\frac{d}{d \xi} F(x, y) / \tau=-\frac{1}{2} e^{-\xi \mu} I_{0}(\xi)
$$

The final result (2.2) now follows by integration. Observe that $\mu$ in (2.12) is larger than unity, and that, hence, the right-hand side of (2.12) tends to zero, when $\xi \rightarrow \infty$. 
Remark 2.1. We derived (2.2) under the assumption $x<y$. As remarked earlier, we can interprete (2.2) also in the limit $x=y$. It easily follows that $F(x, y)$ is continuous (and analytic) on the diagonal $x=y$.

Remark 2.2. Interpreting (2.7) as an inverse Laplace transform, we obtain the known result

$$
\int_{0}^{\infty} e^{-y s} I_{0}(2 \sqrt{t y}) d y=s^{-1} e^{t / s}
$$

Using this with $s=1$ in (1.1), we obtain

$$
I(x, \infty)=\int_{0}^{x} d t=x, \quad I(\infty, y)=y .
$$

Remark 2.3. The path of integration $|s|=\rho=\sqrt{x / y}$ in (2.9) is the so-called saddle-point contour, on which $\operatorname{Im}(x / s+y s)=0$. There are saddle points at $s= \pm \rho$, of which $s=\rho$ is dominant. Observe that the saddle point $s=\rho$ and the pole $s=1$ coincide when $x=y$. This predicts for the uniform asymptotic expansion an error function as approximant.

Remark 2.4. By expanding in (2.9) $1 /(1-s)=\sum s^{m},|s|<1$, a series expansion in terms of modified Bessel functions is obtained. Such expansions appear in Lassey [6].

2.2. A More General Function. Lassey [6] also considered, among others, the function

$$
L(x, y, p)=(1-p) \int_{0}^{x} \int_{0}^{y} e^{-u-t} I_{0}(2 \sqrt{p u t}) d u d t, \quad p \geqslant 0
$$

which is not a true generalization of $(1.1)$, owing to the factor $(1-p)$.

However, $I(x, y)=-\partial L / \partial p($ at $p=1)$. On the other hand,

$$
L(x, y, p)=\left(1-e^{p y-y}\right)+e^{p y-y} K(p y, x)-e^{p x-x} K(y, p x),
$$

which is given in Lassey [6], and can be verified in the same way as (2.4). It follows from (2.10) that in (2.14) $K$ can be replaced by $F$. For (2.14) the limit $p=1$ requires some care, especially when we ask for the computation of $L(x, y, p) /(1-p)$ when $|p-1|$ is small.

3. Uniform Asymptotic Expansion. The expansion of this section can be found in Goldstein [4], where it is written in terms of Whittaker functions. Luke [7] cites this expansion in a more transparent notation. We rederive the expansion and we write, just as Luke, the functions in terms of incomplete gamma functions. Goldstein's starting point for the expansion is a variant version of (2.11). Our method is based on the integral in (2.2). In this way, upper bounds for the remainders are rather easily constructed.

We substitute in (2.2) the well-known expansion

$$
f(t) \sim \frac{1}{\sqrt{2 \pi t}} \sum_{s=0}^{\infty}(-1)^{s} \frac{A_{s}}{t^{s}} \quad \text { as } t \rightarrow \infty,
$$

with

$$
\begin{array}{ll}
A_{s}=\frac{2^{-s} \Gamma\left(\frac{1}{2}+s\right)}{s ! \Gamma\left(\frac{1}{2}-s\right)}, & s=0,1,2, \ldots, \\
A_{s+1}=-\frac{(2 s+1)^{2}}{8(s+1)} A_{s}, & s=1,2, \ldots,
\end{array}
$$


and obtain the formal expansion

$$
F(x, y) \sim \frac{\sqrt{x}+\sqrt{y}}{2 \sqrt{2 \pi \xi}} \sum_{s=0}^{\infty}(-1)^{s} A_{s} \phi_{s},
$$

where

$$
\phi_{s}=\sqrt{\sigma} \int_{\xi}^{\infty} e^{-\sigma t} t^{-s-1 / 2} d t=\sigma^{s} \Gamma\left(\frac{1}{2}-s, \sigma \xi\right)
$$

Here, $\Gamma(a, z)$ is the incomplete gamma function, usually defined as

$$
\Gamma(a, z)=\int_{z}^{\infty} e^{-t} t^{a-1} d t
$$

which for $a=\frac{1}{2}$ reduces to the error function. We have $\Gamma\left(\frac{1}{2}, x^{2}\right)=\sqrt{\pi} \operatorname{erfc} x, x \geqslant 0$, where

$$
\operatorname{erfc} x=\frac{2}{\sqrt{\pi}} \int_{x}^{\infty} e^{-t^{2}} d t
$$

Therefore, as a first approximation, we have

$$
F(x, y) \sim \frac{\sqrt{x}+\sqrt{y}}{2 \sqrt{2 \pi \xi}} \phi_{0}=\frac{\sqrt{x}+\sqrt{y}}{2 \sqrt{2 \xi}} \operatorname{erfc}(\sqrt{y}-\sqrt{x}) .
$$

Further terms are obtained from the recursion

$$
\Gamma(a+1, z)=a \Gamma(a, z)+z^{a} e^{-z},
$$

giving, for the first few terms in (3.3),

$$
\begin{cases}A_{0}=1, & \phi_{0}=\sqrt{\pi} \operatorname{erfc} \sqrt{z} \\ A_{1}=-\frac{1}{8}, & \phi_{1}=2 \sigma\left[\frac{e^{-z}}{\sqrt{z}}-\phi_{0}\right] \\ A_{2}=\frac{9}{128}, & \phi_{2}=\frac{2 \sigma^{2}}{3}\left[2 \phi_{0}+\frac{e^{-z}}{z \sqrt{z}}(1-2 z)\right],\end{cases}
$$

with $z=\sigma \xi=(\sqrt{y}-\sqrt{x})^{2}$. Observe that a uniform bound for $\phi_{s}$ follows from (3.4) by replacing $e^{-\sigma t}$ by $e^{-\sigma \xi}$ :

$$
\phi_{s} \leqslant \frac{\sqrt{\sigma} e^{-\sigma \xi}}{\left(s-\frac{1}{2}\right) \xi^{s-1 / 2}}, \quad s=1,2, \ldots .
$$

From Luke [8, p. 201], we obtain for $z>0$

$$
\frac{\sqrt{z}}{z+s+1 / 2} \frac{e^{-z}}{\xi^{s}}<\phi_{s}<\frac{1}{\sqrt{z}} \frac{z+1}{z+s+3 / 2} \frac{e^{-z}}{\xi^{s}}
$$

where $s=0,1,2, \ldots$. These inequalities show the asymptotic nature of (3.3). More about this is considered in the next section.

The representations for $\phi_{1}$ and $\phi_{2}$ in (3.9) are not stable for large $z$. In fact, early terms in the well-known asymptotic expansion

$$
\phi_{0}=\sqrt{\pi} \operatorname{erfc} \sqrt{z} \sim \frac{e^{-z}}{\sqrt{z}}\left(1-\frac{1}{2 z}+\frac{3}{4 z^{2}}+\cdots\right)
$$


will cancel other contributions in, say, $\phi_{2}$. To describe this in more detail, it is convenient to introduce $r_{s}, c_{s}$ by writing

$$
\phi_{s}=\frac{e^{-z}}{\sqrt{z}} \frac{\sigma^{s} r_{s}}{c_{s}}, \quad c_{s}=(-1)^{s} \frac{\Gamma\left(s+\frac{1}{2}\right)}{\Gamma\left(\frac{1}{2}\right)} .
$$

Then (3.4) and (3.8) give the recursion

$$
r_{s+1}=r_{s}-c_{s} z^{-s}, \quad s=0,1, \ldots
$$

The numbers $c_{s}$ are the coefficients in the expansion (3.12), and $r_{s}$ are the remainders. That is,

$$
r_{0}=\sqrt{\pi z} e^{z} \operatorname{erfc} \sqrt{z}=\sum_{s=0}^{n-1} \frac{c_{s}}{z^{s}}+r_{n}, \quad n=0,1, \ldots,
$$

where, for $n=0$, the empty sum is zero. For large values of $z$, we have

$$
r_{s}-\sum_{j=s}^{\infty} \frac{c_{j}}{z^{j}}, \quad s=0,1,2, \ldots
$$

Therefore, when we use (3.14) for large $z$, we subtract the dominant term in the series in (3.16). For more about this numerical instability, see Section 5.

4. Error Bounds for Remainders. From Olver [9, p. 269] it follows that (3.1) can be replaced by the exact representation

(4.1) $f(t)=\frac{1}{\sqrt{2 \pi t}}\left\{\sum_{s=0}^{n-1}(-1)^{s} \frac{A_{s}}{t^{s}}+\delta_{n} \frac{A_{n}}{t^{n}}\right\}-\frac{i e^{-2 t}}{\sqrt{2 \pi t}}\left\{\sum_{s=0}^{m-1} \frac{A_{s}}{t^{s}}+\gamma_{m} \frac{A_{m}}{t^{m}}\right\}$,

where $\delta_{n}$ and $\gamma_{m}$, for any $t>0$, are bounded as follows:

$$
\left|\delta_{n}\right| \leqslant 2 \chi(n) e^{\pi /(8 t)}, \quad\left|\gamma_{m}\right| \leqslant 2 e^{1 /(4 t)}
$$

with

$$
\chi(n)=\sqrt{\pi} \Gamma\left(1+\frac{1}{2} n\right) / \Gamma\left(\frac{1}{2}+\frac{1}{2} n\right) .
$$

Substitution of (4.1) in (2.2) gives an exact version of (3.3), complete with remainders.

We use the second series in (4.1) with $m=0$. It follows that $F(x, y)$ in (2.2) can be written as

$$
F(x, y)=\frac{\sqrt{x}+\sqrt{y}}{2 \sqrt{2 \pi \xi}}\left[\sum_{s=0}^{n-1}(-1)^{s} A_{s} \phi_{s}+\varepsilon_{n}^{(1)}+\varepsilon_{0}^{(2)}\right],
$$

where $\varepsilon_{n}^{(1)}, \varepsilon_{0}^{(2)}$ are estimated by

$$
\left|\varepsilon_{n}^{(1)}\right| \leqslant 2 \chi(n)\left|A_{n}\right| \phi_{n} e^{\pi /(8 \xi)}, \quad\left|\varepsilon_{0}^{(2)}\right| \leqslant \frac{2 \sqrt{\pi \sigma} e^{1 /(4 \xi)}}{\sqrt{\sigma+2}} \operatorname{erfc} \sqrt{\xi(\sigma+2)} .
$$

Now, since the argument of the error function is bounded away from zero (it equals $\sqrt{x}+\sqrt{y}$ ) when $\xi$ is large, we can use the bound

$$
\operatorname{erfc} z<e^{-z^{2}} / z \quad(z>0) \text {. }
$$

When we compare $\left|\varepsilon_{0}^{(2)}\right|$ with the first term of the series in (4.4), we obtain

$$
\left|\varepsilon_{0}^{(2)} / \phi_{0}\right| \leqslant 2 \sqrt{\pi} \frac{z+\frac{1}{2}}{z+\xi} e^{1 /(4 \xi)-2 \xi}, \quad \xi>0
$$


where we used the lower bound in (3.11). We assume that $\xi$ is large enough to make the upper bound in (4.6) negligible.

The bound for $\varepsilon_{n}^{(1)}$ in (4.5) contains the quantity $\chi(n)$. By Stirling's formula it follows that

$$
\chi(n)=O\left[(\pi n / 2)^{1 / 2}\right] \text { as } n \rightarrow \infty .
$$

A simple upper bound follows from the beta integral for $\chi(n)$ (see Olver [9, p. 118]), written in the form

$$
\chi(n)=\frac{n+1}{2} \int_{0}^{\infty} e^{-(n+1) t / 2} t^{-1 / 2}\left(\frac{e^{t}-1}{t}\right)^{-1 / 2} d t
$$

Using $e^{t}-1 \geqslant t(t \geqslant 0)$, we obtain $\chi(n) \leqslant \sqrt{\pi(n+1) / 2}, n>-1$. We conclude that $\left|\varepsilon_{n}^{(1)}\right| \leqslant \sqrt{2 \pi(n+1)} \exp [\pi /(8 \xi)]\left|A_{n}\right| \phi_{n}$. In other words: the remainder $\varepsilon_{n}^{(1)}$ is bounded by the absolute value of the term with $s=n$ in (3.3), apart from the factor $\sqrt{2 \pi(n+1)} \exp [\pi /(8 \xi)]$. This gives a very simple criterion for terminating the asymptotic expansion (3.3). Observe that a priori bounds of $\left|\varepsilon_{n}^{(1)} / \phi_{0}\right|$ follow from (3.11). We have

$$
\left|\varepsilon_{n}^{(1)} / \phi_{0}\right| \leqslant \frac{\sqrt{2 \pi(n+1)}}{\xi^{n}} e^{\pi /(8 \xi)} \frac{(z+1 / 2)(z+1)}{z(z+n+3 / 2)}
$$

which is not sharp for $z \rightarrow 0$, however.

The above analysis shows that (3.3) is a uniform asymptotic expansion of $F(x, y)$, with $\xi$ as large parameter and $\sigma \in[0, \infty)$ as a uniformity parameter.

5. On the Recursion of the Incomplete Gamma Function. In (3.3) the incomplete gamma functions are needed, which can be obtained by the recursion (3.8). As remarked earlier, the terms $\phi_{s}$ shown in (3.9) exhibit some kind of instability property. For higher terms, this instability becomes more pronounced. Although higher terms are required with less numerical precision than the earlier ones, it is appropriate to pay attention to this matter.

The origin of the instability is (3.8). Observe that we use this relation in backward direction, with negative $a$-values. Gautschi [2] considered the computation of exponential integrals, which are special cases of $\Gamma(a, x)$ with $a=-n$; in the present case we have $a=-n+1 / 2, n=0,1, \ldots$. From Gautschi's analysis, we obtain the following procedure: To compute

$$
\left\{\phi_{s}\right\}_{s=0}^{n}, \quad \phi_{s} \text { defined in (3.4), }
$$

where $n$ is appreciably larger than $z=\sigma \xi$ and $z$ is larger than five, the subsets

$$
\left\{\phi_{s}\right\}_{s=0}^{s_{0}}, \quad\left\{\phi_{s}\right\}_{s=s_{0}}^{n}, \quad s_{0}=[z],
$$

can be computed by recursion with $\phi_{s_{0}}$ as starting value in, respectively, backward and forward direction. See Amos [1] for an implementation of this procedure for computing exponential integrals. Gautschi's approach is also considered in Van Der Laan and Temme [5], with modifications of the error analysis. 
To investigate the need of such a procedure, we consider (3.14). For this recursion, the relative amplification factors $\rho_{s}$ introduced in Gautschi [2] are given by $\rho_{s}=$ $\left|r_{0} / r_{s}\right|$. Suppose that we start with initial value $r_{0}$ having a relative error $\varepsilon_{0}$. Then, according to Gautschi's theory, the quantities $r_{s}$ are generated with relative errors $\varepsilon_{s}=\rho_{s} \varepsilon_{0}$. The $r_{s}$ are not of interest, but the terms $A_{s} \phi_{s}$ in (4.4) are. By normalizing, using (3.13), we consider the summation of

$$
\sum_{s=0}^{n-1}(-1)^{s} A_{s} \phi_{s} / \phi_{0}=\sum_{s=0}^{n-1}(-1)^{s} A_{s} \sigma^{s} r_{s} /\left(c_{s} r_{0}\right) \text {. }
$$

Since $r_{s}$ has relative error $\varepsilon_{s}=\rho_{s} \varepsilon_{0}$, the $s$ th term of the sum has absolute error $\delta_{s}=A_{s} \sigma^{s} \varepsilon_{0} / c_{s}$. From (3.10), (3.11) it follows that the sum in (5.3) equals $1+O(1 / \xi)$, as $\xi \rightarrow \infty$. Consequently, after adding the $s$ th term in (5.3), the $s$ th partial sum has an absolute error $\delta_{0}+\cdots+\delta_{s}$. Since

$$
\frac{A_{s}}{c_{s}}=\frac{2^{-s} \Gamma\left(s+\frac{1}{2}\right)}{s ! \Gamma\left(\frac{1}{2}\right)} \sim \frac{2^{-s}}{\sqrt{\pi s}} \quad \text { as } s \rightarrow \infty,
$$

we claim that summation of the series in (4.4), where the terms are obtained by forward recursion, is a numerically safe procedure, as long as $0 \leqslant \sigma \leqslant 2$.

The above analysis is confirmed in numerical experiments. It appeared that the value $\sigma=2$ is not very critical, especially when $n$ in (4.4) is not too large. For $\sigma=1(1) 10, n=5(1) 15, \xi=5(5) 30$, we computed

$$
S_{n}^{(F)}=\sum_{s=0}^{n-1}(-1)^{s} A_{s} \phi_{s}^{(F)}, \quad S_{n}^{(B)}=\sum_{s=0}^{n-1}(-1)^{s} A_{s} \phi_{s}^{(B)},
$$

where $\left\{\phi_{s}^{(F)}\right\},\left\{\phi_{s}^{(B)}\right\}$ are computed by recursion in, respectively, forward and backward/forward direction; the latter according to the procedure described in (5.2). The starting values $\phi_{0}^{(F)}$ and $\phi_{s_{0}}^{(B)}$ were computed with relative accuracy $10^{-10}$ by using the procedures described in Gautschi [3]. For $\sigma=1,2,3$ and the above values of $n$ and $\xi$, all values of $\left|\left(S_{n}^{(F)}-S_{n}^{(B)}\right) / S_{n}^{(B)}\right|$ were less than $10^{-10}$. For $\sigma \geqslant 4$ values larger than $10^{-10}$ occurred only for $n \geqslant 7$, especially for the larger $\sigma$-values and the smaller $\xi$-values. Our aim was not to compute $F(x, y)$ with relative accuracy $10^{-10}$. Only the instability of the forward recursion was investigated.

To see the consequences for the $(x, y)$-plane, we note that pairs $(x, y)$ defined by the relation $\sigma=$ constant are located on the line

$$
y=\left[2 \sigma^{2}+4 \sigma+1+2(\sigma+1) \sqrt{\sigma(\sigma+2)}\right] x .
$$

For $\sigma=2$ (the maximal value for forward recursion), we obtain $y=(17+12 \sqrt{2}) x$ $\simeq 33.97 x$. It follows that forward recursion can be used for $(x, y)$-pairs satisfying $1 \leqslant y / x \leqslant 33.97$.

It is also important to have a rough idea for which values of $\xi$ the asymptotic expansion (3.3) can be used. From (3.2), (3.10), (3.11) it follows that $\left|A_{s} \phi_{s}\right| \simeq$ $\left|A_{s+1} \phi_{s+1}\right|$ when $s \simeq[2 \xi]$. Therefore, the smallest term in (3.3) occurs at $s \simeq[2 \xi]$. For this value of $s$, we have $\left|A_{s} \phi_{s} / \phi_{0}\right| \simeq \exp (-s) \simeq \exp (-2 \xi)$ (apart from algebraic factors or constants). Consequently, when $\exp (-2 \xi)$ is smaller than the demanded relative precision, $\xi=2 \sqrt{x y}$ is large enough (roughly speaking) to use the asymptotic expansion. Observe that (4.6) also contains $\exp (-2 \xi)$ as dominant part. 
Acknowledgment. I am grateful to the referees and the editor for their constructive comments on an earlier version of the paper.

Centre for Mathematics and Computer Science

P. O. Box 4079

1009 AB Amsterdam, The Netherlands

1. D. E. Amos, “Computation of exponential integrals," ACM Trans. Math. Software, v. 6, 1980, pp. $365-377$.

2. W. GautsChI, "Recursive computation of certain integrals," J. Assoc. Comput. Mach., v. 8, 1961, pp. $21-40$.

3. W. GaUtSCHI, “A computational procedure for incomplete gamma functions," ACM Trans. Math. Software, v. 5, 1979, pp. 466-481.

4. S. Goldstein, "On the mathematics of exchange processes in fixed columns. I. Mathematical solutions and asymptotic expansions," Proc. Roy. Soc. London Ser. A., v. 219, 1953, pp. 151-171.

5. C. G. VAN Der LAAN \& N. M. TEMme, Calculation of Special Functions: The Gamma Function, the Exponential Integrals and Error-like Functions, CWI Tract 10, Centre for Mathematics and Computer Science, Amsterdam, 1984.

6. K. R. LASSEY, "On the computation of certain integrals containing the modified Bessel function $I_{0}(\xi)$," Math. Comp., v. 39, 1984, pp. 625-637.

7. Y. L. LUkE, Integrals of Bessel Functions, McGraw-Hill, New York, 1962.

8. Y. L. LUKE, The Special Functions and Their Approximations, Vol. II, Academic Press, New York, 1969.

9. F. W. J. Olver, Asymptotics and Special Functions, Academic Press, New York, 1974. 